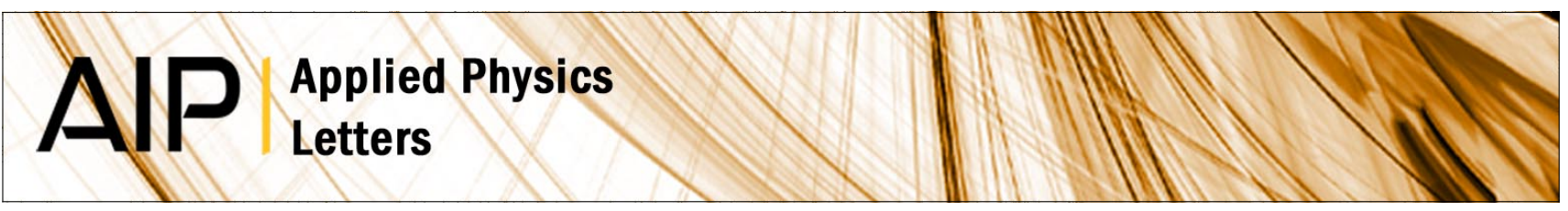

\title{
Nanoscale spin wave valve and phase shifter
}

Y. Au, M. Dvornik, O. Dmytriiev, and V. V. Kruglyak

Citation: Appl. Phys. Lett. 100, 172408 (2012); doi: 10.1063/1.4705289

View online: http://dx.doi.org/10.1063/1.4705289

View Table of Contents: http://apl.aip.org/resource/1/APPLAB/v100/i17

Published by the American Institute of Physics.

\section{Related Articles}

Magnetization dynamics of a MgO-based spin-torque oscillator with a perpendicular polarizer layer and a planar free layer

J. Appl. Phys. 112, 083907 (2012)

Ferrite film growth on semiconductor substrates towards microwave and millimeter wave integrated circuits App. Phys. Rev. 2012, 9 (2012)

Ferrite film growth on semiconductor substrates towards microwave and millimeter wave integrated circuits J. Appl. Phys. 112, 081101 (2012)

Observation of spin-polarized electron transport in Alq3 by using a low work function metal

Appl. Phys. Lett. 101, 102412 (2012)

Dynamic state switching in nonlinear multiferroic cantilevers

Appl. Phys. Lett. 101, 043506 (2012)

\section{Additional information on Appl. Phys. Lett.}

Journal Homepage: http://apl.aip.org/

Journal Information: http://apl.aip.org/about/about_the_journal

Top downloads: http://apl.aip.org/features/most_downloaded

Information for Authors: http://apl.aip.org/authors

\section{ADVERTISEMENT}
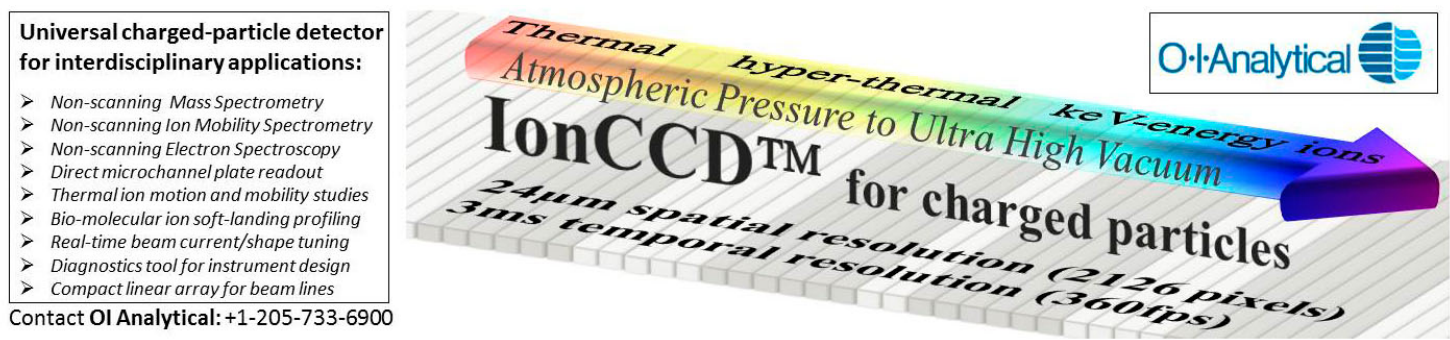


\title{
Nanoscale spin wave valve and phase shifter
}

\author{
Y. Au, ${ }^{\text {a) }}$ M. Dvornik, O. Dmytriiev, and V. V. Kruglyak \\ School of Physics, University of Exeter, Stocker Road, Exeter EX4 4QL, United Kingdom
}

(Received 15 February 2012; accepted 4 April 2012; published online 24 April 2012)

\begin{abstract}
We have used micromagnetic simulations to demonstrate a method for controlling the amplitude and phase of spin waves propagating inside a magnonic waveguide. The method employs a nanomagnet formed on top of a magnonic waveguide. The function of the proposed device is controlled by defining the static magnetization direction of the nanomagnet. The result is a valve or phase shifter for spin waves, acting as the carrier of information for computation or data processing within the emerging spin wave logic architectures of magnonics. The proposed concept offers such technically important benefits as energy efficiency, non-volatility, and miniaturization. (C) 2012 American Institute of Physics. [http://dx.doi.org/10.1063/1.4705289]
\end{abstract}

The short wavelength of spin waves propagating in nanoscale magnonic waveguides at microwave frequencies coinciding with nowadays communication standards could potentially provide a device miniaturization opportunity via accomplishing certain tasks in a more efficient manner when compared to semiconductor circuitry. ${ }^{1,2}$ For example, spin waves have been proposed to be utilized in $\mathrm{GHz}$ filters, ${ }^{3}$ logic devices, ${ }^{4,5}$ amplifiers, ${ }^{6-8}$ mixers, ${ }^{9}$ etc. The utilization of spin waves, within the paradigm of so called "magnonics," is also strongly relevant to exciting such phenomena recently observed in adjacent disciplines as spin transfer torque oscillations, ${ }^{10,11}$ current induced domain wall motion, ${ }^{12}$ spin Hall effect, ${ }^{13}$ spin Seebeck effects, ${ }^{14}$ etc. In light of the burgeoning research in magnonics and related topics, the spin wave technologies are expected to provide a competitive alternative route for performing tasks that are currently accomplished by semiconductor electronics. However, one of the important challenges on the way to fulfillment of this promise of magnonics is to learn to manipulate the spin wave phase and perhaps even magnitude, with this manipulation itself being highly relevant to data encoding and logical operation.

Historically, several spin wave phase shifting mechanisms have been proposed. These include the use of domain walls, ${ }^{15}$ magnetic material nonuniformity, ${ }^{16}$ local electrical voltage, ${ }^{2}$ and flow of electrical current. ${ }^{5,17}$ However, the flow of electrical current produces Joule heating and consumes electrical power. The feasibility of use of piezoelectric elements to control spin waves is yet to be demonstrated while the nucleation of domain wall inside a uniformly magnetized waveguide is energetically costly. At the same time, we have recently demonstrated efficient conversion of a global spatially uniform microwave field into a spin wave propagating in a magnonic waveguide by a reservoir continuous film ${ }^{18}$ and then by a stripe transducer element placed on top of the waveguide. ${ }^{19}$ In this Letter, we demonstrate that a mechanism that is reciprocal to the latter method of spin wave emission ${ }^{19}$ can be used to provide controllable spin wave absorption or phase shift. In particular, a spin wave travelling inside the waveguide resonantly excites the

a) Electronic mail: y.au@exeter.ac.uk. nanomagnet (passive in this case). The excited nanomagnet provides a back action on the incident spin wave, either absorbing its energy or shifting its phase. The action depends upon the static magnetization of the nanomagnet. In particular, the absorption or phase shifting behavior disappears as soon as the static magnetization direction is flipped. We argue that the observed effects could allow one to build a spin wave valve or a spin wave phase shifter, with the static magnetization of the nanomagnet used as an on and off switch for the valve or phase shifter function.

Fig. 1(a) shows the ground state at zero applied magnetic field of a nanomagnet ("resonator") placed on the top of a magnonic waveguide, both made of Permalloy. ${ }^{20}$ The simulations are performed using object oriented micromagnetic framework (OOMMF) (Ref. 21) with the cell size of $5 \times 5 \times 5 \mathrm{~nm}^{3}$. The waveguide is $2200 \mathrm{~nm} \mathrm{long,} 100 \mathrm{~nm}$ wide, and $10 \mathrm{~nm}$ thick, while the resonator is $150 \mathrm{~nm}$ long, $50 \mathrm{~nm}$ wide, and $30 \mathrm{~nm}$ thick. Damping factor $\alpha$ is set to 0.005 for both the waveguide and resonator. The ground state is prepared by relaxing the system under zero applied field $\left(H_{\text {ext }}=0 \mathrm{Oe}\right)$ from a state at which the waveguide and resonator are uniformly magnetized in positive $x$ and $y$ directions, respectively. The gray area on the right hand side of the waveguide depicts the part of the waveguide $\left(50 \times 100 \times 10 \mathrm{~nm}^{3}\right.$ in $x, y, z$ directions, respectively) subjected to a rf magnetic field oscillating at $11.5 \mathrm{GHz}$, with amplitude of $1 \mathrm{Oe}$ and polarized in $y$ direction. The field excites two spin waves with wavelength of about $100 \mathrm{~nm}$ propagating in the waveguide away from the excitation source towards positive and negative $\mathrm{x}$ directions, as shown in Figs. (b)-1(d). The rf frequency $(11.5 \mathrm{GHz})$ is chosen so as to coincide with that of the uniform precession (fundamental mode) of the resonator, deduced from a separate pulse excitation simulation. The damping near the two ends of the waveguide is increased to 0.05 to prevent spin wave reflection. The vertical separation between the resonator and waveguide (in $z$ direction) is 5, 20, and $50 \mathrm{~nm}$ in Figs. 1(b)-1(d), respectively. The incident spin wave drives the resonator into resonance. In turn, the reverse action from the precessing resonator upon the incident spin wave alters its propagation in the waveguide, in a manner that depends strongly on the resonator-waveguide separation. For 


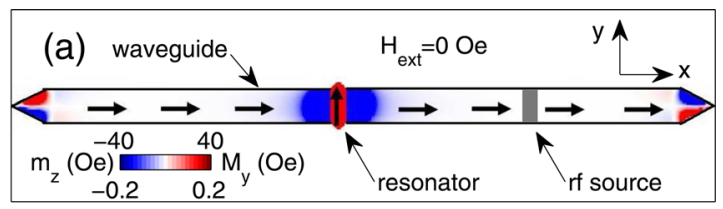

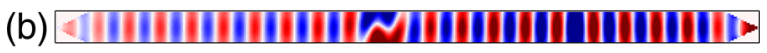

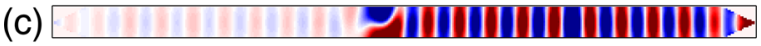

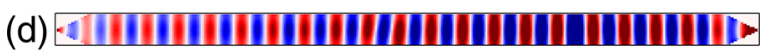

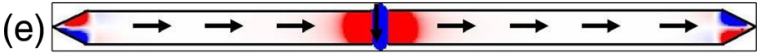

(f)

FIG. 1. (a) Ground state of the micromagnetic structure considered. The entire box of view in (a) represents a $2200 \times 600 \mathrm{~nm}$ region. The magnonic waveguide of $100 \mathrm{~nm}$ width and of $10 \mathrm{~nm}$ thickness is separated by $5 \mathrm{~nm}$ spacing from the overlaid $50 \mathrm{~nm}$ wide, $150 \mathrm{~nm}$ long, and $30 \mathrm{~nm}$ thick resonator. Little arrows inside the waveguide and transducer represent local magnetic moment direction. (b)-(d) Out of plane magnetization $\left(\mathrm{m}_{\mathrm{z}}\right)$ inside the waveguide (static background subtracted) at the same relative simulation time for a vertical spacing between the resonator and the waveguide kept at $5 \mathrm{~nm}$ and changed to 20 and $50 \mathrm{~nm}$, respectively. These images were recorded after the system has attained dynamic steady state. Inset of (a): Color scale for $\mathrm{M}_{\mathrm{y}}$ in (a) and $m_{\mathrm{z}}$ in (b)-(d) with range of -40 to $40 \mathrm{Oe}$ and -0.2 to $0.2 \mathrm{Oe}$, respectively. (e) Ground state of the same structure but with the resonator magnetization flipped to the opposite direction. (f) Waveguide $\mathrm{m}_{\mathrm{z}}$ for case of opposite magnetized resonator at the same aforementioned relative simulation time. Resonator-waveguide spacing equals $5 \mathrm{~nm}$ both in (e) and (f).

example, the spin wave in Fig. 1(b) undergoes an almost 180 degree phase shift while the phase of the spin wave in Fig. 1(d) is largely unaffected. Moreover, for an appropriately set value of the resonator-waveguide separation $(20 \mathrm{~nm}$ in this particular case), the spin wave in the waveguide is predominantly absorbed by the resonator, as demonstrated in Fig. $1(\mathrm{c})$.

The observed phenomena could be highly useful for magnonic applications since their occurrence depends heavily on the static magnetization of the resonator. In particular, the magnetization points towards positive $y$ in Figs. 1(b)-1(d). Fig. 1(e) shows the ground state of the same device but with the magnetization of the resonator toggled to negative $y$ direction. In this case, the resonator does not couple at all to the spin wave propagating in the waveguide, so that its amplitude and phase remain largely unaffected (Fig. 1(f)). Hence, by changing the static magnetization direction of the resonator, it is possible to control the amplitude and phase of the spin wave propagating under the resonator. Depending on the vertical separation between the resonator and the waveguide, these switchable, non-volatile devices could serve as either spin wave valves or phase shifters.

Since the absorption and phase shifting effects arise due to the resonant excitation of the resonator, the time required to bring the excited resonator into steady state of precession would largely define the transient time duration required by the valve or phase shifter to react to change in resonator static magnetization, i.e., operation speed of the device. This "reaction time" in turn is determined mainly by the damping factor of the resonator's material, which has to be artificially adjusted (e.g., by doping) to improve the device performance. Hence, we investigate the device absorption and phase shifting effects as a function of Gilbert damping factor $\alpha$ of the resonator and of the vertical separation between the resonator and the waveguide, while keeping damping factor $\alpha$ of the waveguide at 0.005 as before.

Figs. 2(a) and 2(b) display the relative magnitude of spin wave transmitted in the waveguide under the resonator as measured at $200 \mathrm{~nm}$ away from the central axis of the resonator to negative $x$ direction, for the static magnetization of the resonator pointing towards positive and negative y direction, respectively. The displayed "relative magnitude" is normalized to that measured at the same position on the waveguide in a separate simulation (not shown) that is identical to that discussed but with the resonator removed ("unperturbed case"). In Fig. 2(a), we observe a dark diagonal belt representing absorption maxima that run towards lower values of vertical separation between the resonator and waveguide ("spacing") as $\alpha$ increases. When the static magnetization in the resonator is flipped, the belt largely disappears, as shown in Fig. 2(b). The situation becomes clearer if we inspect the relative phase (again, referenced to the unperturbed case) of the transmitted spin wave, depicted in Fig. 2(c). The phase shift is close to zero on one side of the dark belt of Fig. 2(a), while it is almost 180 degrees on the other side. Again this phase shift phenomenon disappears once the static magnetization of the resonator is flipped (Fig. 2(d)).

We interpret the observations by assuming that the excited resonator emits a spin wave with a phase that is shifted relative to that of the incident spin wave by 180 degrees. The interference between the incident and emitted waves then determines the amplitude and phase of the transmitted wave. Let us consider the case of $\alpha=0.005$ as an example. For small values of the resonator-waveguide
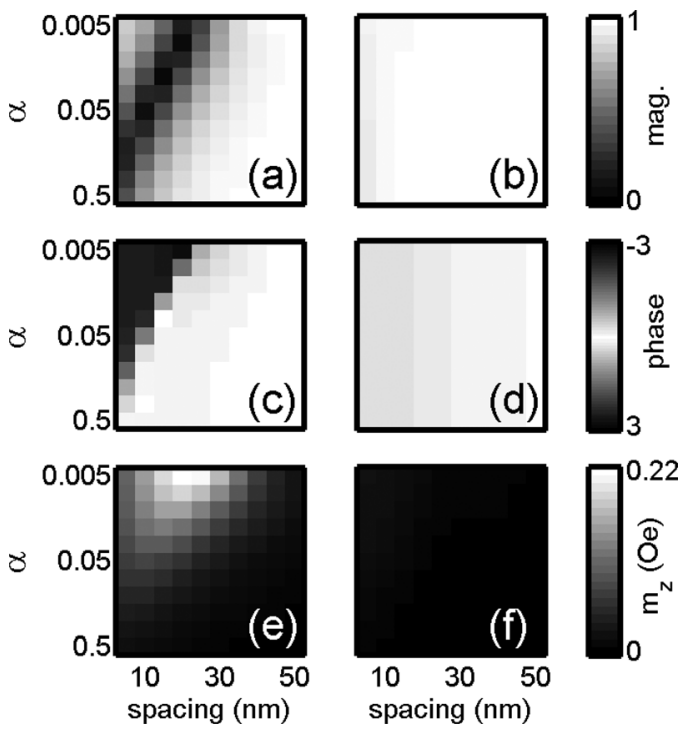

FIG. 2. (a) and (b): Phase diagram of relative magnitude of the spin wave transmitted underneath the resonator measured at $200 \mathrm{~nm}$ in negative $\mathrm{x}$ direction away from the central axis of the resonator for static magnetization of the resonator pointing towards positive and negative y direction respectively. Note that $\alpha$ is plotted in logarithmic scale. (c) and (d): Identical to (a) and (b) but with magnitude of the spin wave replaced by oscillation phase (in radian). (e) and (f): Phase diagram of the precession magnitude of the resonator (averaged over the resonator volume) for static magnetization of the resonator pointing towards positive and negative $y$ direction, respectively. 
spacing, the transmitted wave is dominated by the resonator emitted wave. Indeed, the emitted spin wave results from the dynamic dipolar stray field of the resonator, which is strong if the resonator is positioned near to the waveguide. Hence, the transmitted spin wave demonstrates a 180 degree phase shift.

For large values of the resonator-waveguide spacing, the influence of the resonator stray field on the waveguide diminishes, and so, the incident wave dominates. Hence, the transmitted wave has a zero phase-shift. However, at spacing of $20 \mathrm{~nm}$, the effects of the incident and emitted waves become equal, and so, a complete destructive interference occurs, leading to the observed minimized transmission. As the damping factor of the resonator increases, the minimum moves to smaller values of the resonator-waveguide spacing because the rise of $\alpha$ suppresses the precession amplitude in the resonator. Consequently, the resonator has to be placed nearer to the waveguide in order to compensate the weakening of its stray field and to achieve the same complete destructive interference between the incident and emitted waves. This interpretation is further supported by the dependence of the amplitude of precession in the resonator upon $\alpha$ and spacing, as depicted in Figs. 2(e) and 2(f).

In order to account for the startling difference of the resonator's behavior for the opposite directions of its static magnetization (positive and negative y), we plot in Figures 3(a) to 3(d) the dynamic dipolar stay field of the resonator in $\mathrm{X}-\mathrm{z}$ plane across the middle of the resonator for the relative simulation time of $0,0.125,0.25$, and $0.375 \mathrm{~T}$, respectively, where period $\mathrm{T}$ is equal $0.087 \mathrm{~ns}$. The static magnetization of the resonator points towards positive $y$, and the waveguide is removed for simplicity. If we pay attention to the region
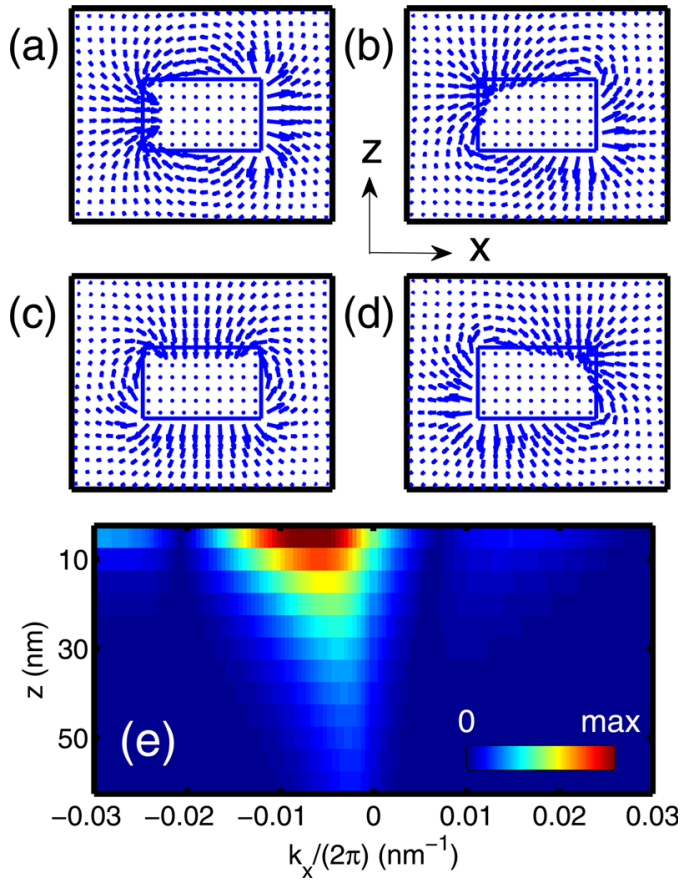

FIG. 3. (a)-(d) Dipolar stray field of the resonator at relative simulation time equal $0,0.125,0.25$, and $0.375 \mathrm{~T}(\mathrm{~T}=1 / 11.5 \mathrm{~ns})$, respectively. (e) Spatial Fourier transform along $\mathrm{x}$ direction of the stray field $\mathrm{z}$ component at different vertical distance from the lower surface of the resonator (vertical axis, z). below the resonator, we observe a pattern of the dipolar field that starts from the right and moves towards left from (a) to (d), echoing a wave travelling towards negative $x$ direction. As the static magnetization inside the waveguide points largely towards positive $x$, the magnetization in the waveguide will only be sensitive to the $z$ component of the resonator's stray field $\left(h_{z}\right)$. The situation becomes even clearer if we Fourier transform $h_{z}$ along $x$ direction, as shown in Fig. 3(e). The Fourier transform displays a striking imbalance between positive and negative $k_{x}$ values, with the majority of the Fourier amplitude residing in the negative region of $k_{x}$. Hence, the resonator is capable of interacting with the spin wave travelling only in one direction (negative $x$ in this case) but not the other (positive $x$ ). When the static magnetization of the resonator is flipped, the chirality of rotation of the resonator's dipolar stray field also flips. This in turn leads to a mirror flip of the Fourier transform of $h_{z}$ in Fig. 3(e) relative to the $k_{x}=0$ axis, thereby completely modifying the effect of the resonator upon the spin wave in the waveguide.

In summary, we have proposed a method by which to achieve magnetically switchable absorption or phase shifting of spin waves travelling in a magnonic waveguide. The mechanism, which relies solely on the state of the static magnetization of a nanomagnet resonator, exhibits advantages of low energy consumption and non-volatility against other mechanisms relying on continuous application of electrical current or voltage. ${ }^{2,5,17}$ The direct current induced domain wall motion ${ }^{12}$ and related spintronic phenomena intensively studied in recent years can become natural methods to provide the static magnetization switching of resonator required in our proposed concept. The concept acts on exchange dominated spin waves in backward volume mode geometry. The latter geometry is preferred for building magnonic circuitry because of its ability to operate at remanence. Furthermore, spin waves in this geometry are able to follow the physical bending of the magnonic waveguide, ${ }^{5,22}$ while the shortness of the spin wave wavelength offers an opportunity to miniaturize magnonic devices. The functionality of the device relies only on a single nanomagnet and is therefore spatially very compact. We have explained the engineering prospects of the concept and have revealed the physical origin of the phenomenon by analyzing the dependence of the wave vector Fourier spectrum of the stray field produced by the resonator. The discovered effects could help to build switchable bandpass filters or even serve as means to control the synchronization of spin transfer torque nano-oscillators. We believe that the proposed devices could provide the foundation for the emerging magnonic nanotechnology.

The research leading to these results has received funding from the EC's Seventh Framework Programme (FP7/ 2007-2013) under GAs 233552 (DYNAMAG) and 228673 (MAGNONICS) and from the EPSRC of the UK (EP/ E055087/1).

${ }^{1}$ V. V. Kruglyak, S. O. Demokritov, and D. Grundler, J. Phys. D: Appl. Phys. 43, 264001 (2010).

${ }^{2}$ A. Khitun and K. L. Wang, J. Appl. Phys. 110, 034306 (2011).

${ }^{3}$ S. K. Kim, K. S. Lee, and D. S. Han, Appl. Phys. Lett. 95, 082507 (2009).

${ }^{4}$ T. Schneider, A. A. Serga, B. Leven, B. Hillebrands, R. L. Stamps, and M.

P. Kostylev, Appl. Phys. Lett. 92, 022505 (2008). 
${ }^{5}$ K. S. Lee and S. K. Kim, J. Appl. Phys. 104, 053909 (2008).

${ }^{6}$ M. Bao, A. Khitun, Y. Wu, J. Y. Lee, K. L. Wang, and A. P. Jacob, Appl. Phys. Lett. 93, 072509 (2008).

${ }^{7}$ A. Khitun, D. E. Nikonov, and K. L. Wang, J. Appl. Phys. 106, 123909 (2009).

${ }^{8}$ E. Padron-Hernandez, A. Azevedo, and S. M. Rezende, Phys. Rev. Lett. 107, 197203 (2011).

${ }^{9}$ Y. Khivintsev, J. Marsh, V. Zagorodnii, I. Harward, J. Lovejoy, P. Krivosik, R. E. Camley, and Z. Celinski, Appl. Phys. Lett. 98, 042505 (2011).

${ }^{10}$ V. E. Demidov, S. Urazhdin, and S. O. Demokritov, Nature Mater. 9, 984 (2010).

${ }^{11}$ M. Madami, S. Bonetti, G. Consolo, S. Tacchi, G. Carlotti, G. Gubbiotti, F. B. Mancoff, M. A. Yar, and J. Akerman, Nature Nanotechnol. 6, 635 (2011).

${ }^{12}$ S. S. P. Parkin, M. Hayashi, and L. Thomas, Science 320, 190 (2008).

${ }^{13}$ Y. Kajiwara, K. Harii, S. Takahashi, J. Ohe, K. Uchida, M. Mizuguchi, H. Umezawa, H. Kawai, K. Ando, K. Takanashi, S. Maekawa, and E. Saitoh, Nature 464, 262 (2010).
${ }^{14}$ K. Uchida, S. Takahashi, K. Harii, J. Ieda, W. Koshibae, K. Ando, S. Maekawa, and E. Saitoh, Nature 455, 778 (2008).

${ }^{15}$ R. Hertel, W. Wulfhekel, and J. Kirschner, Phys. Rev. Lett. 93, 257202 (2004).

${ }^{16}$ S. V. Vasiliev, V. V. Kruglyak, M. L. Sokolvskii, and A. N. Kuchko, J. Appl. Phys. 101, 113919 (2007).

${ }^{17}$ V. E. Demidov, S. Urazhdin, and S. O. Demokritov, Appl. Phys. Lett 95, 262509 (2009).

${ }^{18}$ Y. Au, T. Davison, E. Ahmad, P. S. Keatley, R. J. Hicken, and V. V. Kruglyak, Appl. Phys. Lett. 98, 122506 (2011).

${ }^{19}$ Y. Au, E. Ahmad, O. Dmytriiev, M. Dvornik, T. Davison, and V. V. Kruglyak, "Resonant microwave-to-spin-wave transducer" (unpublished).

${ }^{20}$ Saturation magnetization $M_{\mathrm{s}}=800 \mathrm{Oe}$, exchange stiffness $A=1.3 \times 10^{-11}$ $\mathrm{J} / \mathrm{m}$, and zero magnetocrystalline anisotropy.

${ }^{21}$ M. Donahue and D. G. Porter, oommF User's guide, Version 1.0, Interagency Report NISTIR 6376, NIST, Gaithersburg, MD, 1999.

${ }^{22}$ S. Bance, T. Schrefl, G. Hrkac, A. Goncharov, D. A. Allwood, and J. Dean, J. Appl. Phys. 103, 07 E735 (2008). 